\title{
EXACT COHERENT STRUCTURES IN TURBULENT SHEAR FLOWS
}

\author{
Fabian Waleffe \\ Departments of Mathematics and Engineering Physics \\ University of Wisconsin, Madison, WI 53706, USA \\ waleffe@math.wisc.edu
}

\begin{abstract}
Exact coherent structures are three-dimensional, nonlinear traveling wave solutions of the Navier-Stokes equations. These solutions are typically unstable from onset, yet they capture the basic statistical and structural features of low Reynolds number turbulent shear flows remarkably well. These exact coherent structures have now been found in all canonical shear flows: plane Couette, Poiseuille and pipe flow. They are generic for shear flows and exist for both no-slip and stress boundary conditions. Their discovery opens up new avenues for turbulence research and forces a fundamental rethinking of the true nature of turbulence.
\end{abstract}

\section{Introduction}

What is 'Turbulence'? Is it the random interaction of 'eddies'? That is indeed the prevailing view, motivated on the one hand by the kinetic theory of gases where gases are modeled as the random collisions of point molecules, and on the other hand by one's first impression of turbulent flows: they do look very disordered and 'random.' And they do help mixing milk and coffee.

So the basic model of turbulence is that it merely enhances molecular diffusion. The molecular viscosity $\nu$ is augmented by an eddy or turbulent viscosity $\nu_{T}=\ell_{T} v_{T}$ which is the product of a characteristic or 'mixing length' $\ell_{T}$ and a characteristic velocity $v_{T}$ (see e.g. [23]). Various models specify how to prescribe $v_{T}$ and $\ell_{T}$. In that point of view, homogeneous, isotropic turbulence appears as the fundamental problem and the local turbulent kinetic energy $K(\boldsymbol{x}, t)$ and energy dissipation rate $\varepsilon(\boldsymbol{x}, t)$ as the fundamental quantities of interests. Not surprisingly these models typically perform poorly, if not catastrophically, in strongly inhomogeneous flows, and in particular near walls. Other models abandon the eddy viscosity concept and seek to model directly the Reynolds stresses. But the underlying model of turbulence as the random interaction of 'eddies' is unchanged. Indeed the basic model in that class is the 'return to isotropy' [23]. 
Again, not surprisingly, these models have big troubles near walls and they must be supplemented with various ad hoc strategies such as 'wall-functions'.[23, 22]

There is a fundamentally different view of turbulence that may go back to Hopf, but has been developed in more recent times by Ed Spiegel, Predrag Cvitanovic and co-workers, primarily in the context of low-order dynamical systems. In this view, turbulence is not the random interaction of 'eddies' but rather the random 'switching' from one unstable periodic solution to another. Cvitanovic and collaborators have developed a quantitative cycle expansion method to calculate average properties of a chaotic system in terms of unstable periodic orbits, with the short period solutions dominating the expansion [6]. We now have some evidence that this point of view may also apply to turbulence in fluids.

That evidence has been slowly accumulating over the last 40 years since the work of Kline et al. on the structure of turbulent boundary layers [16], [21]. In wall-bounded turbulent shear flows, a lot of dynamical activity takes place near the wall in the 'buffer region'. This is the region of maximum 'turbulent energy production' and it is also the region where we find organized structures -primarily wavy streaks and quasi-streamwise vortices. These structures are directly connected with the increased drag. They appear randomly in space and time, but statistically they are always there and individual structures are relatively long lived. The desire to understand the physical mechanisms responsible for these coherent structures has led to the development of a self-sustaining process theory $[26,28]$ which in turn led to the discovery of a broad class of Traveling Wave solutions (TWs) of the Navier-Stokes equations for incompressible flows $[29,30,12,32,8,35]$ and suggested the existence of unstable periodic solutions, some of which have now been calculated explicitly [14, 25]. These traveling wave solutions are so similar to the observed structures, qualitatively and quantitatively, that they have been called Exact Coherent Structures [30].

These ideas and recent developments are reviewed below. We emphasize that the goal of these studies at this time is to elucidate the fundamental nature of fluid turbulence not to construct ad hoc engineering turbulence models for complex real-world applications.

\section{Mathematical framework}

We consider the Navier-Stokes equations for incompressible flow

$$
\begin{aligned}
\partial_{t} \boldsymbol{v}+\boldsymbol{v} \cdot \boldsymbol{\nabla} \boldsymbol{v}+\boldsymbol{\nabla} p & =\frac{1}{R} \nabla^{2} \boldsymbol{v}, \\
\boldsymbol{\nabla} \cdot \boldsymbol{v} & =0
\end{aligned}
$$

for the fluid velocity $\boldsymbol{v}(\boldsymbol{x}, t)$ at point $\boldsymbol{x}$ in three-dimensional euclidean space and time $t \geq 0$. The kinematic pressure $p(\boldsymbol{x}, t)$ is the mechanical pressure that enforces the incompressibility constraint and $R$ is the Reynolds number, a non-dimensional parameter that is essentially an inverse fluid viscosity. We consider shear flows with $x$ corresponding to the flow direction. The geometry is planar in this article, with the flow confined between two infinite parallel 
walls perpendicular to the $y$ direction (channels). The velocity components $\boldsymbol{v}=(u, v, w)$ correspond to the cartesian coordinates $(x, y, z)$. Unit vectors in the corresponding cartesian directions are denoted $\hat{\boldsymbol{x}}, \hat{\boldsymbol{y}}$ and $\hat{\boldsymbol{z}}$. The flow is driven by motion of those walls in plane Couette flow, or by an externally imposed pressure gradient in plane Poiseuille flow. The characteristic length scale is chosen as the half-channel height in Couette flow, and the quarter channel height in plane Poiseuille flow (this choice was made for 'morphing' reasons, see [29, $32]$ ). However, the wall-unit value $R_{\tau}=44.2$ quoted for rigid-free Poiseuille flow is based on the half channel height, which is the full wall-normal scale of the quasi-streamwise vortices for the symmetric class of traveling wave solutions considered in this work. Asymmetric traveling waves are also known to exist [12]. We consider periodic boundary conditions in the wall-parallel directions $x$ and $z$ with periods $L_{x}=2 \pi / \alpha$ and $L_{z}=2 \pi / \gamma$, respectively. Fourier expansions are used in the $x$ and $z$ directions, while a well-conditioned Chebyshev integration approach is used for the wall-normal direction $y$. The reader is referred to [32] for further details of the mathematical and numerical formulations.

\section{Self-sustaining process}

\subsection{Inflectional instabilities and streaks}

The Self-Sustaining Process is a conceptual idealization of a fundamental fluid dynamical process in shear flows. This 3D, nonlinear process appears to be the dominant mechanism to extract energy from a shear flow, whether the shear flow is wall-bounded as in pipes and channels, or unbounded as in mixing layers, jets and wakes. This process extracts energy from the mean shear flow $\bar{u}(y)$, which is maintained by external forcing and the boundary conditions, by redistributing that energy in the cross-stream directions $y$ and $z$ where instabilities of inflectional type come into play.

The simplest example of an inflectional instability is the Kelvin-Helmholtz instability of mixing layers such as the hyperbolic tangent profile $U_{L}(y)=\tanh y$ or the Kolmogorov flow $U_{L}(y)=\sin y$. This is a fundamental and robust instability with growth rates that scale with the shear rate $d U_{L} / d y$ as opposed to weak and delicate Orr-Sommerfeld instabilities whose growth rates scale like $R^{-1 / 3}$ where $R$ is the Reynolds number. Inflectional instabilities lead to the roll-up of vortex sheets and the development of vortex arrays similar to von Karman vortex streets. These vortex sheets can then succomb to $2 \mathrm{D}$ pairing instabilities leading to larger vortices or, perhaps more significant, the vortices can break up as a result of 3D instabilities of elliptical type [15]. But the inflectional instability only arises when the vorticity has a local maximum [7, Rayleigh and Fjortoft's theorems]. In the wall-bounded context, pressure effects resulting from wall impermeabillity can reduce or even prevent that instability even if there is an inflection in the laminar flow. For example, the Kolmorogov flow $U_{L}(y)=\sin y$ with boundary conditions $\hat{\boldsymbol{y}} \cdot \boldsymbol{v}=0$ at $y= \pm \pi / 2$ is linearly stable although there is a strong inflection at $y=0$. This is a well-known ex- 
ample due to Tollmien [7] that 'inflections are not enough'. Furthermore, the canonical wall-bounded laminar shear flows such as plane Couette $U_{L}(y)=y$ and Poiseuille $U_{L}(y)=1-y^{2}$ flows do not have inflections.

The self-sustaining process is the $3 \mathrm{D}$ process that circumvents those purely $2 \mathrm{D}$ limitations and unlocks the potential of inflectional instabilities by redistributing the laminar flow $\hat{\boldsymbol{x}} U_{L}(y)$ into a 'streaky' flow $\hat{\boldsymbol{x}} U(y, z)$ to create large inflections in the wall-parallel, spanwise direction $z$ where wall-blocking does not occur. However, linear instability of spanwise varying flows $U(y, z)$ is not enough since the spanwise variations, a.k.a. the streaks $U(y, z)-\bar{u}(y)$ where $\bar{u}(y)$ is the mean shear obtained by averaging the streamwise velocity over $x$ and $z$, are not sustained by the external forcing and the boundary conditions. Hence an inflectional instability of those streaks is likely to simply destroy the spanwise variation that it is extracting energy from, and therefore accelerate the return of the flow to the $1 \mathrm{D}$ laminar state $U_{L}(y)$, rather than triggering a transition to turbulence. For the $1 \mathrm{D}$ shear $\rightarrow 2 \mathrm{D}$ streaks $\rightarrow 3 \mathrm{D}$ streak instability process to be physically significant for transition and turbulence there has to be a feedback process that leads to the regeneration - or recurrence - of streaks. The Self-Sustaining Process (SSP) is the entire 3D, nonlinear process that includes direct nonlinear regeneration of rolls that maintain the streaks in addition to streak generation and streak instability.

From a physical point of view, the SSP can lead to various types of non-trivial 3D shear flows compatible with the forcing and boundary conditions: turbulent flows, time-periodic flows and traveling wave solutions. There is evidence that this basic process occurs at multi-scales, from near wall scales of the order of 100 wall units to the scale of the pipe or channel. A 4th-order model of the SSP has been proposed and derived from the Navier-Stokes equations by a Galerkin truncation [28]. That model captures some basic characteristics of the SSP and demonstrate that the SSP can lead to steady states or periodic states. Refinements of the models in the form of a 9-mode model have been proposed and such models capture further features of the transition to turbulence $[18,19]$. Spatio-temporal models of the SSP have also been developed [17]. But the basic ingredient of the SSP - the advective redistribution of the mean shear by streamwise rolls - is fundamentally an advection-diffusion process that is not well described by low-order sets of ordinary differential equations.

From a mathematical point of view, there is a parallel self-sustaining process theory whose objectives are to develop a precise asymptotic theory of the self-sustaining process for large Reynolds numbers. The theory is weakly nonlinear about a shear flow that has an $O(1)$ spanwise modulation, $U(y, z)$. This SSP theory is essentially a development of the mean flow-first harmonic theory sketched by Benney [2]. The physical concept of the 'self-sustaining process' was inspired primarily by the cartoons of self-regenerating horseshoe vortices in [1]. In the rest of this paper, we focus primarily on the SSP theory and traveling wave solutions of sinusoidal type. 


\subsection{SSP theory, Traveling waves}

We look for traveling wave solutions $\boldsymbol{v}(\boldsymbol{x}, t)=\boldsymbol{v}(\boldsymbol{x}-\operatorname{ct} \hat{\boldsymbol{x}}, 0)$ of the NavierStokes equations, where $c$ is the constant wave velocity to be determined and $\hat{\boldsymbol{x}}$ is the unit vector in the $x$ direction. The full velocity field $\boldsymbol{v}(\boldsymbol{x}, t)$ is Fourier decomposed in the $x$-direction

$$
\boldsymbol{v}=\boldsymbol{v}_{0}(y, z)+\left(e^{i \alpha \theta} \boldsymbol{v}_{1}(y, z)+c . c .\right)+\cdots
$$

where $\theta=x-c t$ and c.c. or ()$^{*}$ denotes complex conjugate. The Fourier decomposition is far from the entire story however, because the various velocity components have very different scalings in the $R \rightarrow \infty$ limit.

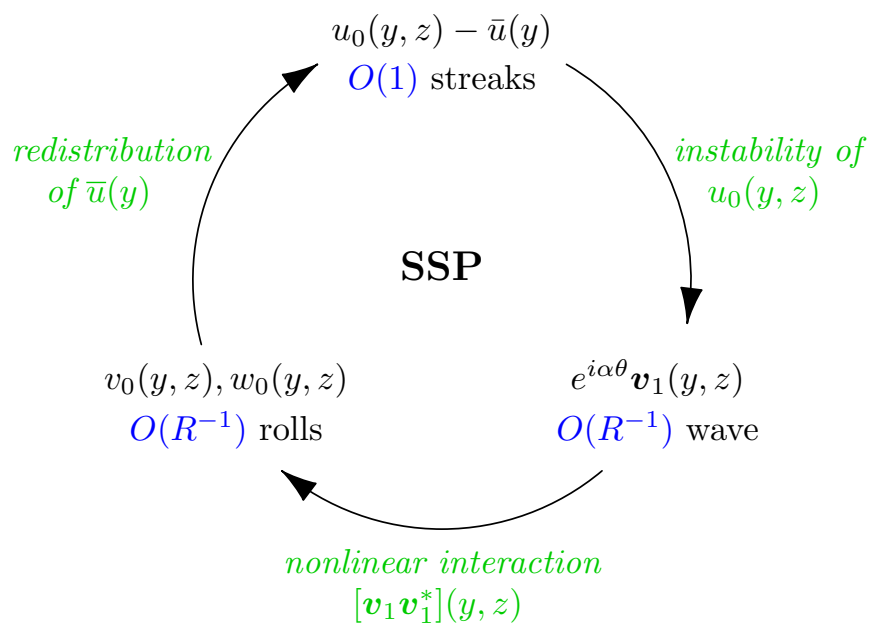

Figure 1: Schematic of the Self-Sustaining Process (SSP) and its asymptotic scaling theory as $R \rightarrow \infty$. The mean shear $\bar{u}(y)$ is maintained by external forcing and the boundary conditions. The rolls $\left(0, v_{0}(y, z), w_{0}(y, z)\right)$, streaks $u_{0}(y, z)-\bar{u}(y)$ and streak wave $e^{i \alpha \theta} \boldsymbol{v}_{1}(y, z)$ maintain each other.

The self-sustaining process theory consists of three main ingredients as illustrated in Figure 1:

1. a 'streaky' flow $u_{0}(y, z) \hat{\boldsymbol{x}}$ that consists of $O(1)$ streaks $u_{0}(y, z)-\bar{u}(y)$ and a mean shear $\bar{u}(y)$ which is the $z$-average of $u_{0}(y, z)$,

2. streamwise rolls $v_{0}(y, z) \hat{\boldsymbol{y}}+w_{0}(y, z) \hat{\boldsymbol{z}}$ of amplitude $O(1 / R)$ that create and sustain the streaks against viscous decay by advective redistribution of the mean $\bar{u}(y)$,

3. a streak wave, $e^{i \alpha \theta} \boldsymbol{v}_{1}(y, z)+$ c.c., which results from an inflectional-type instability of the streaks and whose nonlinear self-interaction $\boldsymbol{v}_{1} \boldsymbol{v}_{1}^{*}$ extracts energy from the streaks $u_{0}(y, z)-\bar{u}(y)$ but sustains the rolls. 
Such a theory had been sketched by Benney [2] and has been developed and linked to near wall coherent structures by the author and collaborators in a series of papers $[9,26,28]$. The spanwise modulation of the streamwise velocity $u_{0}(y, z)-\bar{u}(y)$ are called streaks after the streaky hydrogen bubble patterns that revealed them in the original observations [16]. The streak eigenmode $e^{i \alpha \theta} \boldsymbol{v}_{1}(y, z)+$ c.c. is the first Fourier harmonic in the $x$-direction and has zero $x$ average. Its quadratic nonlinear interaction, $e^{2 i \alpha \theta} \boldsymbol{v}_{1} \boldsymbol{v}_{1}+\boldsymbol{v}_{1} \boldsymbol{v}_{1}^{*}+$ c.c., generates a 2nd harmonic $e^{2 i \alpha \theta} \boldsymbol{v}_{2}(y, z)$ but, more importantly, it generates the key $x$ independent Reynolds stresses that not only extract energy from the streaks but also feedback on the streamwise rolls. This dual role of the Reynolds stresses, where the $\overline{u v}^{x}$ and $\overline{u w}^{x}$ stresses extract energy from the streaks and the $\overline{v v}^{x}$, $\overline{v w}^{x}$ and $\overline{w w}^{x}$ stresses sustain the rolls, requires a 3D perturbation and 3 distinct ingredients: rolls, streaks and streak eigenmode, in addition to the mean shear that provides the overall energy. Here $\overline{(~)}^{x}$ denotes an $x$-average only. Three ingredients are necessary because the streak instability extracts energy from the streaks, hence it cannot directly sustain the streaks, but it can sustain the rolls which in turn sustain the streaks. The entire process is sustained by the mean shear.

This process has been studied and illustrated through low-order modeling [28] as well as analysis of the various elements independently $[9,26,28]$, but more recently it has been used as the basis for a method to construct fully-resolved steady state and traveling wave solutions of the Navier-Stokes equations. The self-sustaining process can appear as a periodic or an equilibrated process. In the periodic version, rolls create streaks whose inflectional instability create an $x$-dependent mode whose nonlinear development recreates the rolls, and these three phases of the process occur in succession. In the equilibrated version of the process, the three elements, rolls, streaks and streak eigenmode, have just the right structure and amplitude to stay in mutually sustained equilibrium. The initial DNS-based studies [33, 9] revealed the periodic version. Later, a controlled bifurcation approach was developed and provided a direct validation of the self-sustaining process theory in fully resolved calculations of the NavierStokes equations.

In that approach, a weak artificial forcing of $O\left(1 / R^{2}\right)$ is added to the NavierStokes equations to sustain steady rolls. This weak forcing acts as vortex generators on an airplane wing. It generates steady rolls of amplitude $O(1 / R)$ which in turn redistribute the streamwise velocity to create steady $O(1)$ streaks. This part of the process - the formation of streaks from streamwise rolls - is linked to the many recent studies of non-normal linear amplification and optimum linear growth which show that streamwise rolls of amplitude $\epsilon / R$ lead to streaks of amplitude $O(\epsilon)$. However in those linear studies (linearized about the laminar flow that is) it is necessary to have $\epsilon \ll 1$ for self-consistency since $\epsilon=O(1)$ is accompanied by a modification of the mean shear of $O(1)$ and therefore a strong departure from the laminar flow and from linear theory about the laminar flow $[27,28]$. The next step in the SSP-based procedure is to detect the parameters that correspond to a neutrally stable streaky flow, either by increasing the roll forcing for a fixed streamwise wavenumber $\alpha$ or by varying $\alpha$ for a fixed roll 
forcing. That neutrally stable point corresponds to a bifurcation point. According to the SSP theory that bifurcation should be subcritical in terms of the roll forcing parametrized by an $O(1)$ parameter $F_{r}$. Therefore, tracking the 3D bifurcating solution to higher amplitudes (the amplitude of the 3D perturbation now becomes the control parameter) should go to lower roll forcing $F_{r}$. If the bifurcating solution can be tracked all the way to $F_{r}=0$, corresponding to no artificial roll forcing, then a 3D self-sustained steady state or traveling wave has been found. This procedure has been used successfully in plane Couette flow with both free-slip (imposed shear) and no-slip (imposed velocity) boundary conditions [29, 32], as well as in channel and pipe flows [8, 35]. It is possible that this approach might also enable the direct construction of some periodic solutions.

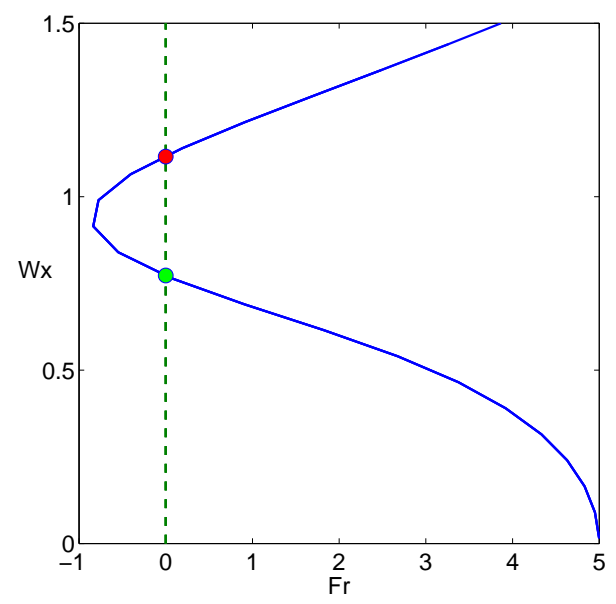

Figure 2: Bifurcation from streaky flow in free-slip plane Couette flow with $(\alpha, \gamma, R)=(0.49,1.5,150) . F_{r}$ is the normalized roll amplitude and $W_{x}$ is the normalized $x$-dependent streak mode amplitude as defined in the text. The $1 \mathrm{D}$ laminar flow is at $(0,0) .\left(F_{r} \neq 0, W_{x}=0\right)$ correspond to $2 \mathrm{D}$ streaky flows. The streaky flow is neutrally stable at $F_{r} \approx 5$. A $3 \mathrm{D}$ steady state solution bifurcates from the streaky flow at that point and reaches all the way to $F_{r}=0$, providing two self-sustained 3D steady states in this free-slip plane Couette flow. The lower branch solution is indicated by the green dot and the upper branch is indicated by the red dot.

The procedure is illustrated in figure 2 for 'free-slip' plane Couette flow. The parameter $F_{r}$ measures the roll forcing, normalized such that the maximum wall-normal velocity of the resulting rolls is $F_{r} / R$. The parameter $W_{x}=$ $R|\bar{\eta}(1,0)|$ corresponds to the $y$-averaged vertical vorticity $(\eta)$ amplitude of the $\left(k_{x}, k_{z}\right)=(1,0)$ Fourier mode times the Reynolds number $R$, where $\left(k_{x}, k_{z}\right)$ are the integer indices of the wavenumbers in the $x$ and $z$ directions respectively. The fundamental spanwise wavenumber is set at $\gamma=1.5$ and the fundamental 
streamwise wavenumber is fixed at $\alpha=0.49$. The laminar flow corresponds to the origin on the plot $\left(F_{r}, W_{x}\right)=(0,0)$. Streaks appear as soon as $F_{r} \neq 0$ but they are not unstable for these values of $(\alpha, \gamma, R)=(0.49,1.5,150)$ until $F_{r} \approx 5$. The streaky flow is unstable for those parameters in the interval $5<F_{r}<18.4$ (approximatively). For $F_{r}<5$ the streaks are too weak to be unstable, and for $F_{r}>18.4$ the rolls are so strong that they completely wipe out the shear flow, transporting momentum between the walls much faster than viscosity can replenish it. The streaks are again very weak, the flow is dominated by the rolls and there is no instability. Continuation of the upper branch on figure 2 leads to that 2 nd bifurcation point at $F_{r} \approx 18.4$.

The upper and lower branch solutions are connected in the self-sustained $F_{r}=0$ parameter space, where they appear through a saddle node bifurcation at a critical Reynolds number $R \approx 142$ for those length scales $(\alpha=0.49, \gamma=1.5)$. Those self-sustained 3D solutions form a two-parameter $(\alpha, \gamma)$ family of plane Couette flow steady states. Likewise, there is a two-parameter family of steady states in rigid-rigid (no-slip) plane Couette flow that first appear through a saddle node bifurcation at $R \approx 127.7$ [32] and there is a two-parameter family of traveling wave solutions in plane Poiseuille flows that first appear at $R_{\tau} \approx 44.2$ $[30,32]$. There are also related but distinct families of other solutions. For instance, there exist asymmetric, 'one-walled' traveling waves in plane Poiseuille flow [12]. The steady state solutions obtained in rigid-rigid plane Couette flow belong to the same family as the solutions computed by Nagata [20] and Clever and Busse $[4,5]$.

This procedure demonstrates the validity of the SSP but also provides a robust method to calculate these non-trivial 3D solutions. Indeed this approach has enabled the discovery of a related family of traveling wave solutions in pipe flow $[8,35,11]$. This construction is necessary because all those solutions are linearly unstable and cannot be obtained by simple direct numerical simulations. But if these solutions are unstable, why should we care? The answer to that question is directly tied to the nature of turbulence. We start by illustrating these solutions and showing the connections with observed coherent structures.

\section{Exact coherent structures}

The steady state (in plane Couette) and traveling wave solutions (in channel and pipe flows) obtained by the SSP-based bifurcation from streaky flow approach are closely related to one another. Indeed when one of those solutions is obtained in the plane geometry, say in free-slip plane Couette, it is easy to obtain related solutions in the plane geometry by 'morphing' or 'homotopy' from one flow to another and/or one type of boundary conditions to another as was initially done in $[29,30,32]$. It is possible, for instance, to morph a free-free plane Couette steady state to a rigid-free plane Poiseuille traveling wave in 10 or fewer Newton steps (as in the Rayleigh-Bénard literature, 'rigid' refers to no-slip and 'free' to free-slip (imposed stress) boundary conditions, so 'rigid-free' means no-slip on the bottom wall and free-slip on the top wall). All these solutions have the 
same basic structure, they consists of wavy streaks flanked by staggered, quasistreamwise vortices. These features correspond exactly to the structures that have been observed in the near-wall region of turbulent shear flows.

Qualitative similarity would already by quite interesting since these are nontrivial 3D traveling wave solutions of the Navier-Stokes equations, but there is also quantitative similarity. Indeed, optimizing the traveling wave solutions over the streamwise and spanwise periods in order to determine the smallest friction Reynolds number $R_{\tau}$ at which these solutions exist leads to a minimum value for $R_{\tau}$ of about 44.2. That value is obtained for the length scales $L_{x}^{+} \approx 273.7$ and $L_{z}^{+} \approx 105.5$. Thus $R_{\tau} \approx 44.2$ is the smallest value at which this class of solution exists. There are solutions of this class for larger $R_{\tau}$ 's but not for smaller ones. The friction Reynolds number $R_{\tau}=u_{*} h / \nu$ where $h$ is the halfchannel height and $u_{*}$ is the friction velocity defined in terms of the kinematic viscosity $\nu$ and the shear rate at the wall $d \bar{u} /\left.d y\right|_{w}$ according to $u_{*}^{2}=\nu d \bar{u} /\left.d y\right|_{w}$. All three of those length scales, $L_{x}^{+}, R_{\tau}$ and $L_{z}^{+}$, are essentially identical to the typical length scales for near-wall coherent structures reported in the literature [24]. That optimum solution is illustrated in figure 3 .
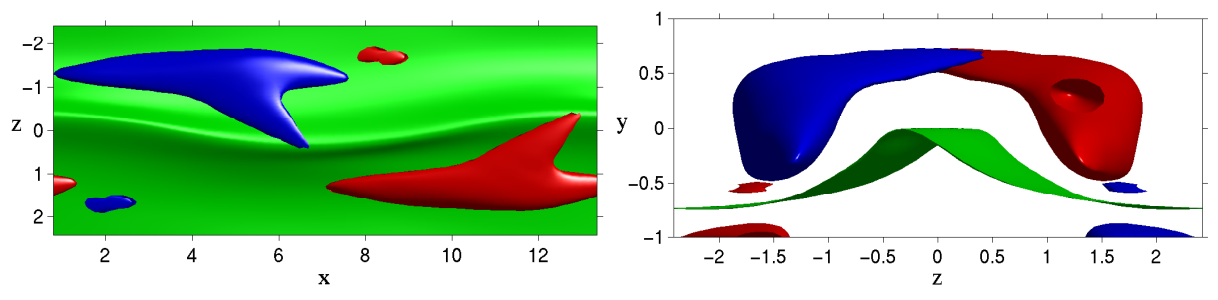

Figure 3: Top view and end view of a traveling wave solution in plane Poiseuille flow (half-channel, the bottom wall is at $y=-1$ and the channel centerline is at $y=+1$ ) at its minimum friction Reynolds number $R_{\tau}=44.21$ obtained for the optimum parameters $\alpha=0.51$ and $\gamma=1.3$ corresponding to $L_{x}^{+}=273.7$ and $L_{z}^{+}=105.5$. The green isosurface of streamwise velocity $u$ illustrates the wavy streaks, the streamwise vorticity $\omega_{x}$ is represented by the red $\left(\omega_{x}>0\right)$ and blue $\left(\omega_{x}<0\right)$ isosurfaces, visualizing the staggered, counter-rotating, quasistreamwise vortices.

\subsection{Upper and Lower branches}

The exact coherent structures - steady state or traveling wave solutions of the Navier-Stokes equations - come in pairs, an upper branch and a lower branch. The upper branch has the highest drag and its drag grows quickly with $R$ (fig. 4). The structure of the upper branch changes with $R$ and it is expected that further bifurcations take place to give rise to multiscale features in order to transport momentum efficiently. We expect that the near wall structures will look similar to the observations and to optimum solutions such as that shown in fig. 3. But larger scales features are necessary to transport momentum efficiently 
away from the wall. The complete solutions might have a multiscale structure reminiscent of the 'multi-alpha' optimum transport field calculated by Busse [3]. The upper branch solutions and their suspected multiple bifurcations are more costly and challenging to compute for higher $R$. The drag of the lower branch is higher than the laminar value but asymptotes to a constant small multiple of the laminar value (the latter corresponds to the drag for the $1 \mathrm{D}$ velocity profile $\boldsymbol{v}=(y, 0,0))$. An $R$ vs. Drag plot is presented in figure 4 for two pairs of fundamental horizontal wavenumbers $(\alpha, \gamma)$. It illustrates the rapid increase with $R$ of the drag associated with the upper branch solutions while the lower branch drag asymptotes to a value about 30 to $40 \%$ higher than laminar.

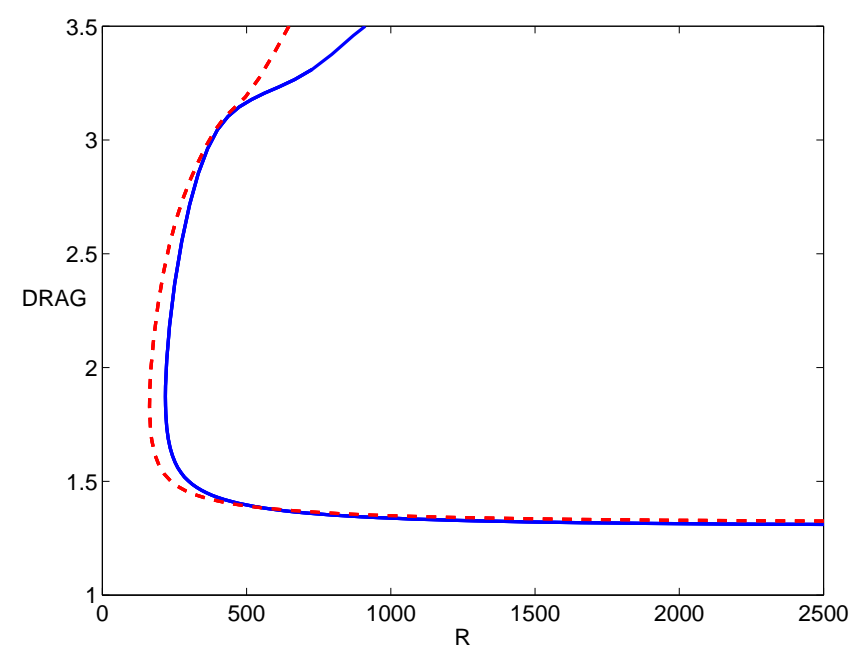

Figure 4: Reynolds number $R$ vs. Drag normalized by its laminar value for rigid-rigid plane Couette flow and $(\alpha, \gamma)=(1.14,2.505)$ (solid), $(1,2)$ (dashed).

An upper branch steady state solution in rigid-rigid plane Couette flow is illustrated in figure 5. That solution is not optimum, in fact it is close to the smallest streamwise length scale for which the steady states exist for that value of the spanwise length scale $L_{z}=2 \pi / \gamma$ with $\gamma=1.67$ and for $R=400$. For smaller values of $L_{x}=2 \pi / \alpha$, the steady states appear to be replaced by periodic solutions $[9,14,31,32]$. The nature of that 'transition' is not understood at this time.

\subsection{Asymptotic structure of the lower branch solutions}

Figure 6 shows a lower branch solution at $(\alpha, \gamma, R)=(1.14,2.5,400)$. Here the vortical motions are illustrated using the $60 \%$ isosurface of $2 Q=\nabla^{2} p$ which is also (twice) the second invariant of the velocity gradient tensor. This visualization technique is not optimum but it successfully separates vortical motions straddling the streaks from the shear layers at the walls, allowing a perspective 


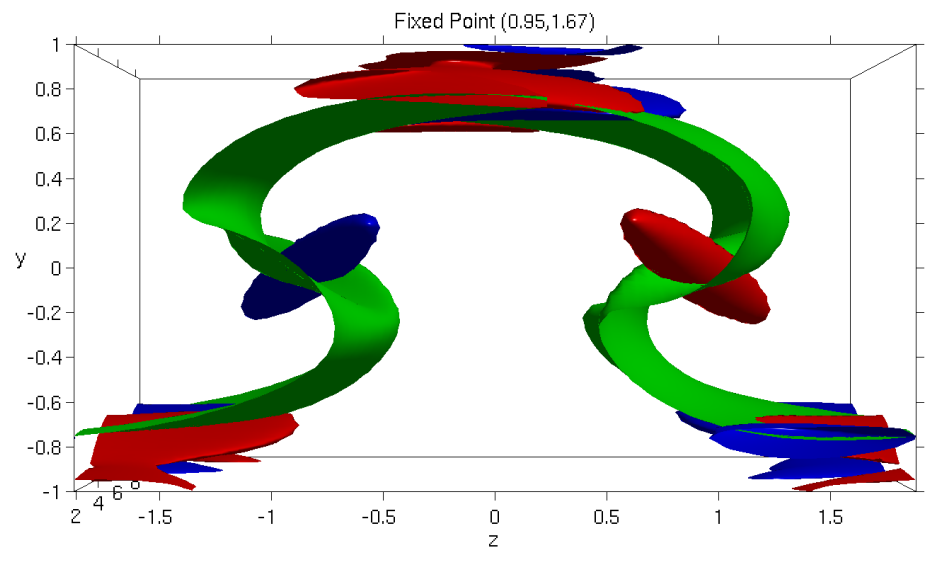

Figure 5: End view of the upper branch steady state solution in rigid-rigid plane Couette flow at $(\alpha, \gamma, R)=(0.95,1.67,400)$. The green isosurface is $u=0$ illustrating the wavy streaks, streamwise vorticity $\omega_{x}$ is represented by the red $\left(\omega_{x}>0\right)$ and blue $\left(\omega_{x}<0\right)$ isosurfaces, visualizing the staggered, counterrotating, quasi-streamwise vortices. The no-slip boundary condition induces shear layers near the walls but these do not correspond to vortices.

view in rigid-rigid plane Couette flow. The upper branch solutions vary substantially with the parameters $(\alpha, \gamma, R)$ but figure 6 is typical of the lower branch solutions. The latter show $O(1)$ streaks (as illustrated by the warped $u=0$ green isosurface) with much weaker streak waviness (undetectable on the plot) and weak quasi-streamwise vortical motions that can hardly be called vortices. The main effect of those vortical motions is a vertical updraft of $O(1 / R)$ at $z=0$ and a corresponding downdraft at $z= \pm L_{z} / 2$. These up and downdrafts sustain the $O(1)$ streaks, in perfect agreement with the SSP theory.

A closer look at such a lower branch solution is given in figures 7 and 8 for $(\alpha, \gamma)=(1.14,2.505)$ and the much higher $R=6192$ in rigid-rigid plane Couette flow. Figure 7 shows the vertical and spanwise velocity component of the $x$-averaged flow, $v_{0}(y, z)$ and $w_{0}(y, z)$, that form the 'streamwise rolls' in the SSP theory. The structure of these streamwise rolls is not as simple as that of a Stokes modes but they do correspond to an updraft at $z=0$ and downdraft at $z= \pm L_{z} / 2$. The thick black line is the contour $u_{0}(y, z)=0$ which is almost identical to $u(x, y, z)=0$ since the flow is dominated by the $u_{0}$ component. Indeed $u_{0}(y, z)$ contains an $O(1)$ mean shear and an $O(1)$ spanwise fluctuation $u_{0}(y, z)-\bar{u}(y)$, with much weaker rolls $\left(v_{0}(y, z), w_{0}(y, z)\right)$ of $O(1 / R)$ and a weak fundamental $x$-undulation $e^{i \alpha x} \boldsymbol{v}_{1}(y, z)$. The scalings of these components has been considered in [34] and further studies will be published elsewhere. The asymptotic scaling of the fundamental undulation $e^{i \alpha x} \boldsymbol{v}_{1}(y, z)+$ c.c. is delicate because that mode has a critical layer structure about $u_{0}(y, z)=0$ as suggested by fig. 8 which shows the real parts of $v_{1}(y, z)$ and $w_{1}(y, z)$ closely hugging 


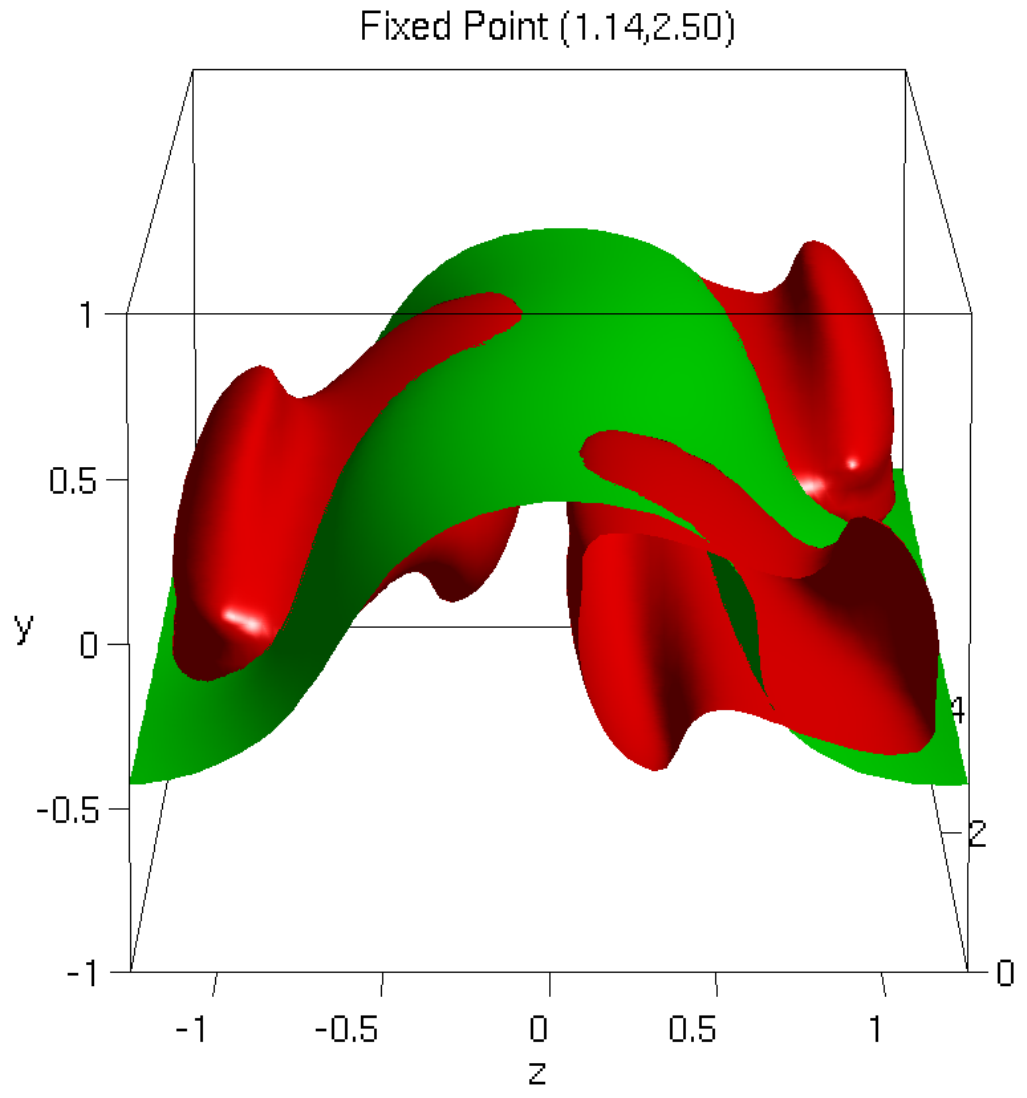

Figure 6: The lower branch solution in rigid-rigid plane Couette flow for $(\alpha, \gamma, R)$ $=(1.14,2.5,400)$. The red isosurfaces are the $60 \%$ level set of $2 Q=\nabla^{2} p$. The surface on the right side corresponds to clockwise vortical motion and that on the left to counter-clockwise vortical motion. Those vortical motions and the waviness of the streaks (undetectable on this plot) are of low amplitudes but the streaks are $O(1)$. This lower branch structure is characteristic of other parameters, including much higher Reynolds number. 

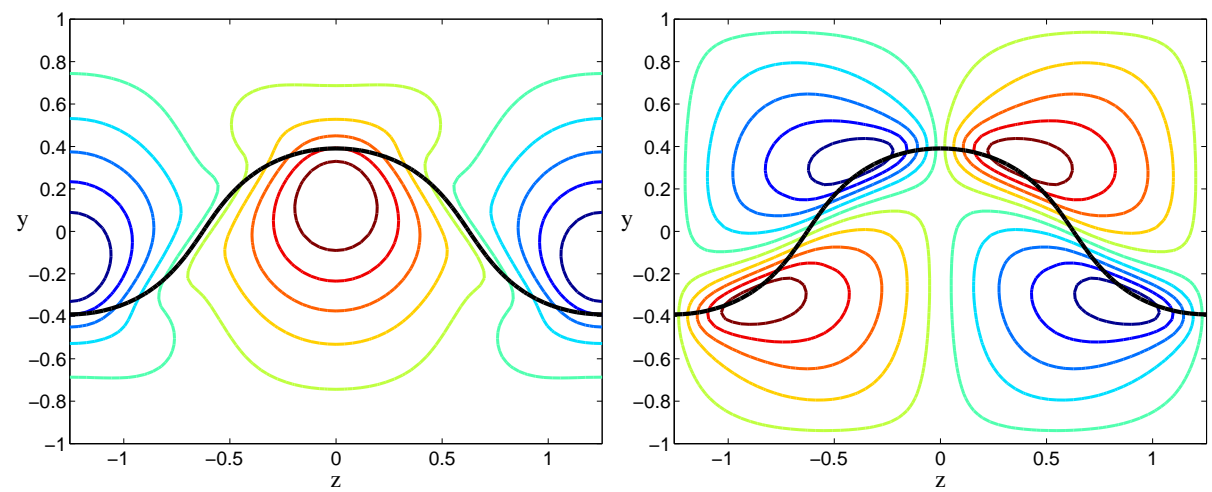

Figure 7: Structure of the streamwise rolls $v_{0}(y, z) \hat{\boldsymbol{y}}+w_{0}(y, z) \hat{\boldsymbol{z}}$ for the lower branch steady state in rigid-rigid plane Couette flow at $(\alpha, \gamma, R)=$ $(1.14,2.505,6196)$. Thick line is the critical layer velocity $u_{0}(y, z)=0$. Left: contours of $v_{0}(y, z)$, right: contours of $w_{0}(y, z)$. Red is positive, blue is negative.

the $u_{0}(y, z)=0$ surface. Our latest studies (asymptotics + numerics up to about $R \approx 60,000$ ) indicate that the critical layer is a classical $R^{-1 / 3}$ layer, however this mode which becomes singular in the limit $R \rightarrow \infty$, is nonlinearly coupled to the rolls that sustain the streaks, so the asymptotic analysis is nontrivial. These asymptotic results are important because they give solid evidence that these steady states exist for all Reynolds numbers - they are not just a low Reynolds number curiosity, and since solutions occur in pairs, existence of the lower branch implies existence of at least one upper branch. The relative simplicity of the lower branch states suggests that a rigorous mathematical proof of their existence might be achievable. The asymptotic results also indicate that these solutions do not bifurcate from the laminar flow, not even at $R=\infty$, since these states tend to a flow that retains streaks of $O(1)$ as $R \rightarrow \infty$. The relatively smooth large scale (shear layer size) structure of these lower branch states, except for a critical layer detached from the walls, also suggests that these solutions will be robust to boundary conditions. They may even exist in the presence of rough boundaries.

\section{Coherence within turbulence}

We have already seen how the exact coherent structures capture some key characteristics of the coherent structures observed in the near-wall region of turbulent shear flows. They consist of wavy streaks flanked with staggered quasistreamwise vortices, and their optimum scaling, in the sense of smallest $R_{\tau}$ at which they exist, is very close to the experimentally reported values. In particular, the minimum wall-unit thickness is about $44.2^{+}$obtained for a streak spacing of about $105.5^{+}$and a streamwise period of about $273.7^{+}$. But there are 

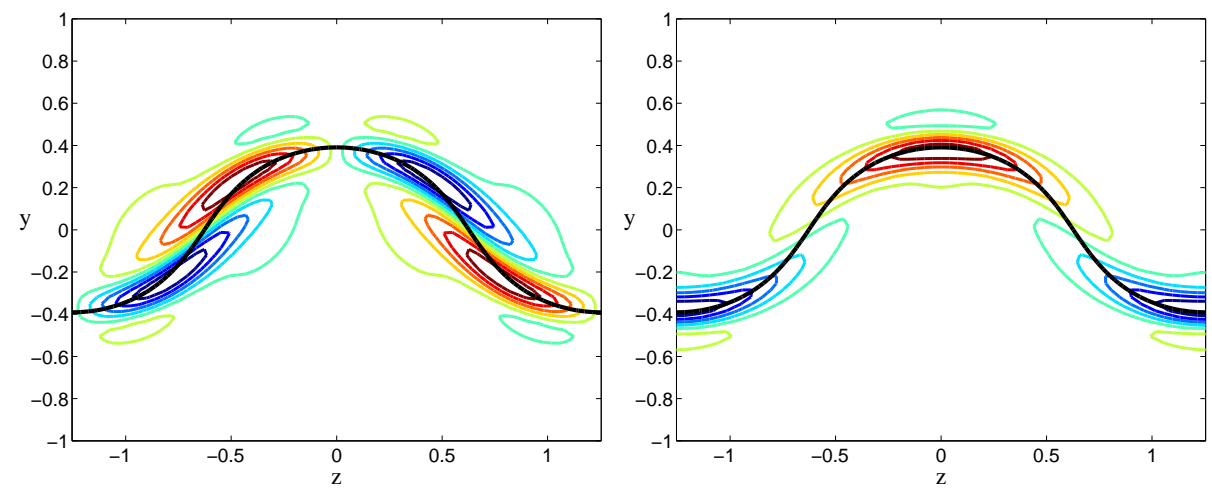

Figure 8: Structure of the streak wave $e^{i \alpha(x-c t)} \boldsymbol{v}_{1}(y, z)$ for the lower branch steady state $(c=0)$ in rigid-rigid plane Couette flow at $(\alpha, \gamma, R)=$ $(1.14,2.505,6196)$. Thick line is the critical layer velocity $u_{0}=0$. Left: $\Re v_{1}(y, z)$, right: $\Re w_{1}(y, z)$.

further connections with turbulence as illustrated in figure 9 which shows the total energy input rate $I$ in rigid-rigid plane Couette flow versus the total energy dissipation rate $D$, both normalized to their laminar value so that $I=D=1$ corresponds to the laminar state. The total energy input and dissipation rates are basic quantities of interest in turbulence theory and this two-dimensional picture of the dynamics was first introduced by Kawahara and Kida in this context [14].

\subsection{Upper branches and Turbulence}

Figure 9, suggests that the upper branch solution, shown by the red marker, forms the 'backbone' or 'organizing center' in phase space for the turbulent 'attractor,' assuming that the latter exists in this domain at this low $R$. The orbit shown in figure 9 was computed for over 2000 convective time units, starting from near the unstable upper branch solutions at numerical resolution $32 \mathrm{x} 49 \mathrm{x}$ $32(x, y z$, Fourier in $x, z$, Chebyshev in $y)$. A higher resolution calculation using $48 \times 65 \times 48$ from the same initial conditions 'patched' to that higher resolution (i.e. with the extra modes set to zero initially) was sustained for over 1000 time units but eventually decayed. Thus the existence of a turbulent attractor in this domain at this $R=400$ is not assured but there are certainly very long-lived turbulent-like states, in the 'neighborhood' of the upper branch solution. The mean and RMS velocity profiles of the 'turbulent' solution calculated at $32 \times 49$ x 32 are remarkably well captured by the upper branch fixed point as shown in figure 10 . 


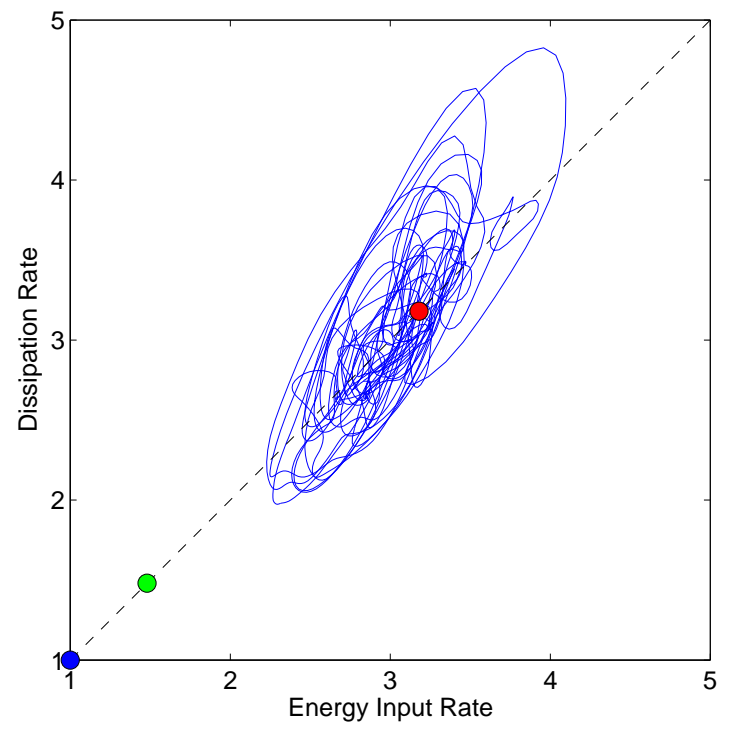

Figure 9: Energy Input vs. Dissipation rate in rigid-rigid plane Couette for $(\alpha, \gamma, R)=(0.95,1.67,400)$. The blue orbit is a 'turbulent' trajectory at this relatively low $R$. The blue dot is the laminar point, the green dot is a lower branch 3D steady state and the red dot is the corresponding upper branch steady state.

\subsection{Lower branches, transition and control}

The presumed phase space importance of the lower branch solutions is that they form the backbone of the separatrix - the phase space boundary between the basin of attraction of the laminar flow and that of the turbulent 'attractor'. The separatrix would consists of the stable manifold of that lower branch state. This simplistic picture is illustrated in figure 11. Similar views were discussed in $[12,32]$ and supported by the data analyses in [13].

Cartoon 11 is probably too simplistic. It is likely that there is more than one lower branch state and there are also lower branch periodic states. Nonetheless, our studies do lend some validity to that simple picture. The lower branch states in rigid-rigid plane Couette flow typically have only one unstable mode, so their unstable manifold is one dimensional. We have verified using Direct Numerical Simulations at the higher $R=1000$ that starting on the 'laminar side' of the stable manifold leads uneventfully back to the laminar flow. The structure remains similar to the lower branch state (fig. 6) but decays slowly. The $x$-structure disappears first, most likely because it has a viscous critical layer structure, and the flow tends to straight rolls and streaks which then slowly (on a viscous time scale) decay together back to the laminar flow. In contrast, starting 'on the other side' of the stable manifold, that is in the other direction on the 1D unstable manifold, rapidly (on a convective time scale) leads to a 

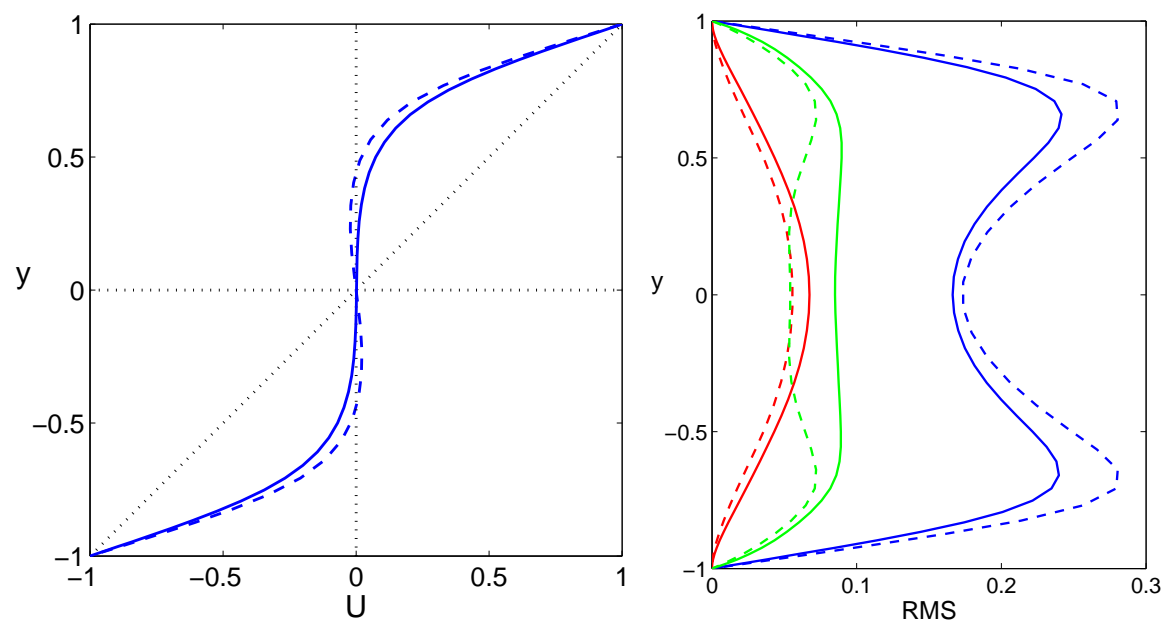

Figure 10: Left: Mean velocity profile in rigid-rigid plane Couette flow at $(\alpha, \gamma, R)=(0.95,1.67,400)$. Right: RMS velocity fluctuation profiles, $u_{r m s}$ in blue, $v_{r m s}$ in red and $w_{r m s}$ in green. Turbulent averages (over 2000 times units) is solid, upper branch fixed point is dashed.

turbulent state. This confirms that the transition threshold question - what is the smallest perturbation of the laminar flow that can trigger turbulence - is a question about the shortest distance from the laminar fixed point to the stable manifold of the lower branch. The SSP theory, which is closely connected to the lower branch, suggests that a good way to trigger turbulence is to start with weak streamwise rolls of $O(1 / R)$, which will lead to $O(1)$ streaks, together with some small $x$-perturbation to trigger the streak instability that will feedback on the rolls thereby approaching the lower branch state. As long as the initial perturbation is slightly on the turbulent side of the separatrix, the flow will approach the lower branch state then 'burst' toward turbulence along the unstable manifold of the lower branch. We emphasize that this 'bursting' is not the linear streak instability, it is the linear instability of the nonlinear $3 \mathrm{D}$ lower branch state. This $O(1 / R)$ scaling as well as the general form of the perturbation is in good agreement with the experimental results of Hof, Juel and Mullin in pipe flow [10].

The low dimensionality ( 1 even at $R=1000$ ) of the unstable manifold of the lower branch suggests that these lower branch states could be good targets for control. Most control strategies aim at either maintaining the linearly stable laminar flow or relaminarizing the fully turbulent flow. The idea here would be to trigger the $3 \mathrm{D}$ lower branch state and stabilize it. These states are relatively smooth and have the scale of the shear layer. They have few and clear modes of linear instability, so the controller knows what instability to control in contrast to the cases of the linearly stable but nonlinearly unstable laminar flow, and of the multiscale turbulent flow. There is a small drag penalty for being on the 


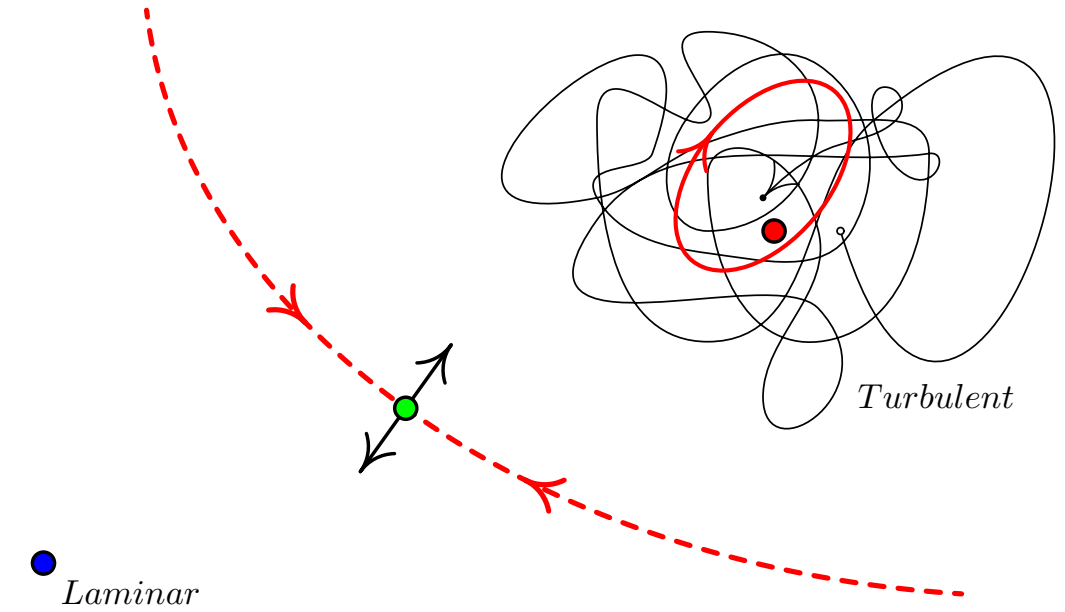

Figure 11: Phase space cartoon of the role of exact coherent states. Blue marker is the laminar fixed point. Black orbit is a turbulent trajectory, red marker is an upper branch solution, green is the lower branch solution. The red dash is the stable manifold of the lower branch, it is the separatrix between the laminar and turbulent basins of attraction. The red orbit is an unstable periodic solution.

lower branch states, but it is only about 30 to $40 \%$ higher than laminar.

\section{$6 \quad$ Not So Final remarks}

Turbulence in shear flows is arguably the most fundamental kind of hydrodynamic turbulence. This is the turbulence that we find when a fluid flows by a wall, in a channel or in a pipe. The recent findings briefly reviewed in this paper are that such flows admit 3D traveling wave solutions that capture and tightly link the primary coherent structures observed in the near wall region of wall bounded turbulent shear flows for over 40 years. These structures consists of wavy streaks flanked by staggered quasi-streamwise vortices and this is exactly the structure of the traveling wave solutions. The agreement is not only qualitative but also quantitative since the smallest scales at which these traveling waves exist matches the experimentally observed dimensions of coherent structures: a streak spacing of about 100 wall units, a wall-normal vortex size of about 50 wall units and a streamwise streak undulation of about 300 wall units. The traveling waves also capture the mean velocity profiles and the RMS fluctuation profiles. But all those traveling wave solutions are unstable. That of course is where 'turbulence' comes in. Since the solutions are unstable, the flow can never settle onto any one of those solutions. However their unstable manifolds are typically quite low-dimensional and there are many similar solutions. Statistically, the flow spends most of its time near one or another equivalent solution, explaining why a single solution upper branch solution can be so good 
at capturing the statistics of turbulent flows. The solutions come in pairs, an upper branch and a lower branch. While the upper branches are the organizing centers for the turbulent attractor, the lower branches are the organizing centers for the boundary separating the basin of attraction of the laminar flow from that of the turbulent attractor. Hence the lower branch solutions are directly related to the questions of transition and prevention of turbulence. This is a view of turbulence that is quite different from the 'random collision of eddies'. In addition to the traveling wave solutions, relative periodic orbits are being extracted from the Navier-Stokes equations [25]. So this is far from the end, the story is just beginning.

\section{Acknowledgements}

The numerical and asymptotic analysis of the lower branch solutions is the focus of Jue Wang's PhD thesis. She produced figures 4, 7, 8 and computed the turbulent orbit in figures 9 and 10. That computation was performed using John Gibson's ChannelFlow code. This research has been partially supported by the US NSF-DMS 0204636. This paper was prepared for the Proceedings of the Conference on Turbulence and Interactions held in Porquerolles, France, May 29-June 2, 2006. I thank the organizers Michel Deville, Pierre Sagaut and Thien-Hiep Le for the opportunity to present this work at that international conference.

\section{References}

[1] M.S. Acarlar and C.R. Smith. A study of hairpin vortices in a laminar boundary layer. J. Fluid Mech., 175:1-41 and 45-83, 1987.

[2] D.J. Benney. The evolution of disturbances in shear flows at high Reynolds numbers. Stud. Appl. Math., 70:1-19, 1984.

[3] F. H. Busse. The optimum theory of turbulence. In Advances in Applied Mechanics. (A79-47538 21-34), volume 18, pages 77-121. New York, Academic Press., 1978.

[4] R.M. Clever and F.H. Busse. Three-dimensional convection in a horizontal fluid layer subjected to constant shear. J. Fluid Mech., 234:511-527, 1992.

[5] R.M. Clever and F.H. Busse. Tertiary and quaternary solutions for plane couette flow. J. Fluid Mech., 344:137-153, 1997.

[6] P. Cvitanović, R. Artuso, R. Mainieri, G. Tanner, and G. Vattay. Chaos: Classical and Quantum. Niels Bohr Institute, Copenhagen, 2005. ChaosBook.org.

[7] P. Drazin and W.H. Reid. Hydrodynamic Stability. Cambridge University Press, Cambridge, UK, 1981. 
[8] H. Faisst and B. Eckhardt. Traveling waves in pipe flow. Phys. Rev. Lett., 91:224502, 2003.

[9] J. Hamilton, J. Kim, and F. Waleffe. Regeneration mechanisms of near-wall turbulence structures. J. Fluid Mech., 287:317-348, 1995.

[10] B. Hof, A. Juel, and T. Mullin. Scaling of the turbulence transition threshold in a pipe. Phys. Rev. Lett., 91:244502, 2003.

[11] Bjorn Hof, Casimir W.H. van Doorne, Jerry Westerweel, Frans T.M. Nieuwstadt, Holger Faisst, Bruno Eckhardt, Hakan Wedin, Richard R. Kerswell, and Fabian Waleffe. Experimental observation of nonlinear traveling waves in turbulent pipe flow. Science, 305(5690):1594-1598, 2004.

[12] T. Itano and S. Toh. The dynamics of bursting process in wall turbulence. J. Phys. Soc. Japan, 70:703-716, 2001.

[13] J. Jimenez, G. Kawahara, M.P. Simens, and M. Nagata. Characterization of near-wall turbulence in terms of equilibrium and 'bursting' solutions. Phys. Fluids, 17:015105 (16pp.), 2005.

[14] G. Kawahara and S. Kida. Periodic motion embedded in Plane Couette turbulence: regeneration cycle and burst. J. Fluid Mech., 449:291-300, 2001.

[15] Richard R. Kerswell. Elliptical instability. Annual Review of Fluid Mechanics, 34(1):83-113, 2002.

[16] S.J. Kline, W.C. Reynolds, F.A. Schraub, and P.W. Rundstadler. The structure of turbulent boundary layers. J. Fluid Mech., 30:741-773, 1967.

[17] P. Manneville. Spots and turbulent domains in a model of transitional plane Couette flow. Theoretical and Computational Fluid Dynamics, 18:169-181, November 2004.

[18] J. Moehlis, H. Faisst, and B. Eckhardt. A low-dimensional model for turbulent shear flows. New Journal of Physics, 6:56+17, 2004.

[19] J. Moehlis, H. Faisst, and B. Eckhardt. Periodic orbits and chaotic sets in a low-dimensional model for shear flows. SIAM J. Applied Dynam. Systems, 4:352-376, 2004.

[20] M. Nagata. Three-dimensional finite-amplitude solutions in plane Couette flow: bifurcation from infinity. J. Fluid Mech., 217:519-527, 1990.

[21] R.L. Panton, editor. Self-Sustaining Mechanisms of Wall Bounded Turbulence. Computational Mechanics Publications, Southampton, 1997.

[22] U. Piomelli and E. Balaras. Wall-layer models for large-eddy simulations. Annual Review of Fluid Mechanics, 34(1):349-374, 2002. 
[23] Stephen B. Pope. Turbulent Flows. Cambridge University Press, Cambridge, UK, 2000.

[24] S K Robinson. Coherent motions in the turbulent boundary layer. Annual Review of Fluid Mechanics, 23(1):601-639, 1991.

[25] Divakar Viswanath. Recurrent motions within plane Couette turbulence. Journal of Fluid Mechanics, 2006 (submitted).

[26] F. Waleffe. Hydrodynamic stability and turbulence: Beyond transients to a self-sustaining process. Stud. Applied Math., 95:319-343, 1995.

[27] F. Waleffe. Transition in shear flows: Nonlinear normality versus nonnormal linearity. Phys. Fluids, 7:3060-3066, 1995.

[28] F. Waleffe. On a self-sustaining process in shear flows. Phys. Fluids, 9:883$900,1997$.

[29] F. Waleffe. Three-dimensional coherent states in plane shear flows. Phys. Rev. Lett., 81:4140-4148, 1998.

[30] F. Waleffe. Exact coherent structures in channel flow. J. Fluid Mech., 435:93-102, 2001.

[31] F. Waleffe. Exact coherent structures and their instabilities: Toward a dynamical-system theory of shear turbulence. In Shigeo Kida, editor, Proceedings of the International Symposium on "Dynamics and Statistics of Coherent Structures in Turbulence: Roles of Elementary Vortices", pages 115-128. National Center of Sciences, Tokyo, Japan, 2002.

[32] F. Waleffe. Homotopy of exact coherent structures in plane shear flows. Phys. Fluids, 15:1517-1543, 2003.

[33] F. Waleffe, J. Kim, and J. Hamilton. On the origin of streaks in turbulent shear flows. In F. Durst, R. Friedrich, B.E. Launder, F.W. Schmidt, U. Schumann, and J.H. Whitelaw, editors, Turbulent Shear Flows 8: selected papers from the Eighth International Symposium on Turbulent Shear Flows, Munich, Germany, Sept. 9-11, 1991, pages 37-49. Springer-Verlag, Berlin, 1993.

[34] F. Waleffe and J. Wang. Transition threshold and the self-sustaining process. In T. Mullin and R.R. Kerswell, editors, Non-uniqueness of Solutions to the Navier-Stokes Equations and their Connection with LaminarTurbulent Transition, pages 85-106. Kluwer, 2005.

[35] H. Wedin and R.R. Kerswell. Exact coherent structures in pipe flow. J. Fluid Mech., 508:333-371, 2004. 